\title{
Nonequilibrium readiness and precision of Gaussian quantum thermometers
}

\author{
Luca Mancino $\odot,{ }^{1}$ Marco G. Genoni, ${ }^{2}$ Marco Barbieri $\odot,{ }^{3,4}$ and Mauro Paternostro ${ }^{1}$ \\ ${ }^{1}$ Centre for Theoretical Atomic, Molecular and Optical Physics, School of Mathematics and Physics, \\ Queen's University, Belfast BT7 1NN, United Kingdom \\ ${ }^{2}$ Quantum Technology Lab, Dipartimento di Fisica, Università degli Studi di Milano, 20133 Milano, Italy \\ ${ }^{3}$ Dipartimento di Scienze, Università degli Studi Roma Tre, Via della Vasca Navale 84, 00146 Rome, Italy \\ ${ }^{4}$ Istituto Nazionale di Ottica, CNR-INO, 50125 Florence, Italy
}

(Received 7 May 2020; accepted 24 August 2020; published 25 September 2020)

\begin{abstract}
The dimensionality of a thermometer is key in the design of quantum thermometry schemes. In general, the phenomenology that is typical of qubit-based quantum thermometry does not apply to infinite-dimensional ones. We analyze the dynamical and metrological features of nonequilibrium Gaussian quantum thermometers: On one hand, we highlight how quantum entanglement can enhance the readiness of composite Gaussian thermometers; on the other hand, we show that nonequilibrium conditions do not guarantee the best sensitivities in temperature estimation, thus suggesting the reassessment of some of the working principles underpinning quantum thermometry.
\end{abstract}

DOI: 10.1103/PhysRevResearch.2.033498

\section{INTRODUCTION}

The direct assessment of the properties of quantum mechanical systems is not always possible or convenient: In general, any direct interference would alter the properties of the system, possibly spoiling them. In addition to such a problem, the need to characterize systems which cannot be directly accessed has fostered the research for effective schemes for indirect quantum probing [1,2]. Quantum thermometry, i.e., the estimation of the operating temperature of a quantum system, offers interesting opportunities for the design and application of indirect probing strategies, which would be useful for the characterization and control of temperature of microand nanodevices [3-7].

Most of the current investigations in quantum thermometry use two-level systems as thermometers [8-11], shedding light on the link between the equilibrium heat capacity of such microscopic probes and the amount of information that can be gathered on the temperature of the environment [12-14], introducing bounds on the irreversible entropy production of the probe [15-17], and clarifying the extent of the advantages resulting from finite-time interactions for both temperature discrimination and estimation [14,18]. Such investigations have ultimately opened the path to the exploration of the role played by genuine quantum features in the enhancement of the thermometric performance of two-level quantum probes [19-29]. However, little has been explored about the effects

Published by the American Physical Society under the terms of the Creative Commons Attribution 4.0 International license. Further distribution of this work must maintain attribution to the author $(s)$ and the published article's title, journal citation, and DOI. that the dimensionality of the quantum probe has on the features of a given thermometric protocol.

This is precisely the context within which the study reported in this article is set. We consider quantum thermometry operated using infinite-dimensional (Gaussian) quantum probes, each interacting with its own thermal bath, whose temperature we aim at estimating [30-32]. We show that entanglement between the probes significantly impacts on how fast the two-mode Gaussian state changes as a consequence of the coupling to the external environment. We call such a rate of change readiness and use it as the core figure of merit of our work. The latter quantifies how fast the two-mode Gaussian state changes as a consequence of the presence of the external environment. Our study marks significant discrepancies with the recently explored qubit-based case reported in Ref. [33], where geometric considerations on the dynamics of the thermometer have been explored.

Here we show that a composite thermometer operating under nonequilibrium conditions does not always offer higher sensitivities in temperature estimation, thus leaving room for the reappraisal of some of the aspects underpinning quantum thermometry $[14,23]$. While the formalism used to illustrate our findings is that of Gaussian quantum states and operations [34-36], our study addresses a wealth of physical situations of strong experimental relevance for quantum probing, from micro-/nanomechanical oscillators driven by optical or electric forces to microwave fields in superconducting waveguides and atomic spin systems collectively coupled to driving fields [37].

The remainder of this work is organized as follows. In Sec. II we describe the model addressed in our analysis. Section III is devoted to geometric considerations leading to the definition of a dynamical speed on the Riemannian manifold of quantum states. In Sec. IV we discuss the metrological precision of the probing mechanism through the evaluation of 


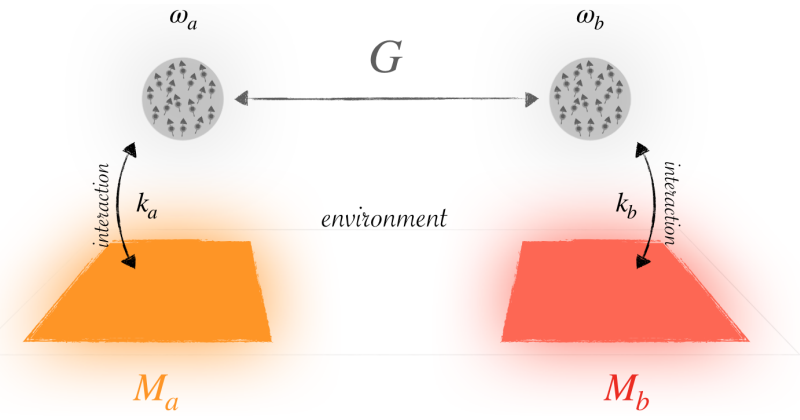

FIG. 1. Conceptual scheme of the protocol. Two ensembles of atomic spin states are led to interact with an environment which is made of two independent Markovian thermal baths at temperatures $T_{a}$ and $T_{b}$, with average numbers of excitations $M_{a}$ and $M_{b}$. The interaction between the two ensembles is modulated through the $G$ parameter. The dissipation to the local thermal bath depends on the $k_{a, b}$ decay rate.

the nonequilibrium quantum Fisher information (QFI) of the system. Section V then summarizes our findings and offers different perspectives of investigation.

\section{PHYSICAL MODEL}

We consider a thermometric scheme which is based on the weak interaction between two $N$-atom spin systems (labeled as $a$ and $b$ ) and their respective thermal baths (see Fig. 1). Collective states of atomic spin systems have long been considered for metrological tasks in light of their high sensitivity [38], and thus embody a natural platform where to investigate thermometry.

The ground and excited states of each atom are $|g\rangle$ and $|e\rangle$, respectively, and the free Hamiltonian of the probing system is $\hat{H}^{0}=\hbar \omega_{a}\left(\sum_{k=1}^{N} \hat{\sigma}_{k, a}^{z}+1 / 2\right)+\hbar \omega_{b}\left(\sum_{k=1}^{N} \hat{\sigma}_{k, b}^{z}+\right.$ $1 / 2)$, where $\omega_{a, b}$ represents the transition frequency of the atoms. The ensembles are coupled through the interaction term

$$
\hat{H}^{\text {int }}=\frac{\hbar G}{2} \hat{S}_{a}^{x} \hat{S}_{b}^{x}
$$

where we have introduced the collective spin operators $\hat{S}_{j}^{x}=$ $\hat{S}_{j}^{-}+\hat{S}_{j}^{+}$with $\hat{S}_{j}^{ \pm}=\sum_{k=1}^{N} \hat{\sigma}_{k, j}^{ \pm}(j=a, b)$ and $\hat{\sigma}_{k}^{+}=\hat{\sigma}_{k}^{-\dagger}=$ $|e\rangle\left\langle\left. g\right|_{k}\right.$. Equation (1) results from the off-resonant dipolelike coupling of the two ensembles with the same light field, which is then adiabatically eliminated [38]. As collective spin systems are symmetric with respect to particle exchange, we can ignore any external degree of freedom associated with the position of the individual particles.

As mentioned, local and independent baths, coupled to the respective subsystems at a rate $k_{j}$, induce thermal fluctuations described through the zero-mean input operators $\hat{j}^{\text {in }}$, each characterized by the two-time correlation functions $\left\langle\hat{j}^{\text {in, }}{ }^{\dagger}(t) \hat{j}^{\text {in }}\left(t^{\prime}\right)\right\rangle=M_{j} \delta\left(t-t^{\prime}\right)$ and $\left\langle\hat{j}^{\text {in }}(t) \hat{j}^{\text {in, }}{ }^{\dagger}\left(t^{\prime}\right)\right\rangle=$ $\left(M_{j}+1\right) \delta\left(t-t^{\prime}\right)$. Here $M_{j}=\left(e^{\hbar \omega_{j} / k_{B} T_{j}}-1\right)^{-1}$ is the thermal occupation number of bath $j$ at temperature $T_{j}$ and $k_{B}$ is the Boltzmann constant [39].
We assume that the spin system exhibits no large fluctuations (enforced by the assumption of low temperature, i.e., $M_{j} \lesssim 1$ ), so the collective spin operators can be mapped onto effective bosonic degree of freedom, with creation and annihilation operators $\hat{j}$ and $\hat{j}^{\dagger}(j=a, b)$, respectively, through a Holstein-Primakoff (HP) transformation [40,41]. In this regime, the interaction Hamiltonian is recast into the form $\hat{H}_{H P}^{\text {int }}=\hbar n_{G} / 2\left(\hat{a}^{\dagger}-\hat{a}^{\dagger} \hat{a}^{\dagger} \hat{a} / 2 N+\right.$ H.c. $)\left(\hat{b}^{\dagger}-\right.$ $\hat{b}^{\dagger} \hat{b}^{\dagger} \hat{b} / 2 N+$ H.c.); in this contribution, terms of order higher than quadratic are suppressed by at least a factor $1 / N$. Further details on the manipulation of the Hamiltonian via the HP transformation are given in Appendix A. From now on, we refer to the total Hamiltonian of the system in the HP regime as $H_{H P}$.

It is convenient to introduce the vector of dimensionless quadrature operators of the system $\hat{f}=\left(\hat{x}_{a}, \hat{p}_{a}, \hat{x}_{b}, \hat{p}_{b}\right)$, where $\hat{x}_{j}=\left(\hat{j}^{\dagger}+\hat{j}\right) / \sqrt{2}$ and $\hat{p}_{j}=i\left(\hat{j}^{\dagger}-\hat{j}\right) / \sqrt{2}$. The dynamics of $\hat{f}$, which can be described using the formalism of HeisenbergLangevin equations, is made very challenging by the presence of nonlinear terms in $H_{H P}$. In order to overcome this hurdle, we consider the fluctuations of the HP operators by taking the first-order expansion $\hat{f}_{j}=\bar{f}_{j}+\delta \hat{f}_{j}$, where $\bar{f}_{j} \in \mathbb{C}$ is a classical mean value and $\delta \hat{f}_{j}$ is a zero-mean fluctuation operator [42]. Such expansion is justified by the assumption of low temperatures and allows for the linearization of the equations of motion, which can be cast in the form

$$
\partial_{t} \delta \hat{f}=A \delta \hat{f}+R^{\text {in }},
$$

with $\hat{R}^{\text {in }}=\left(\sqrt{2 k_{a}} \hat{x}_{a}^{\text {in }}, \sqrt{2 k_{a}} \hat{p}_{a}^{\text {in }}, \sqrt{2 k_{b}} \hat{x}_{b}^{\text {in }}, \sqrt{2 k_{b}} \hat{p}_{b}^{\text {in }}\right)$ (we recall that the input noise operators are already zero-mean quantities). We have introduced the drift matrix

$$
A=\left(\begin{array}{cccc}
-k_{a} & \omega_{a} & 0 & 0 \\
-\omega_{a} & -k_{a} & -n_{G} & 0 \\
0 & 0 & -k_{b} & \omega_{b} \\
-n_{G} & 0 & -\omega_{b} & -k_{b}
\end{array}\right),
$$

with the rescaled coupling strength $n_{G}=N G$. Equation (2) describes the dynamics of the quadrature operators for the two ensembles and includes terms accounting for dissipation at rate $k_{j}$ into the local baths.

The linearity of such equations and the choice of initial Gaussian states imply that the probability distribution describing the system is Gaussian at any time. Under these conditions, the complete description of the whole system can be enshrined in the covariance matrix $\sigma(t)$ of the fluctuations, whose entries are defined $\sigma_{m n}(t)=\left\langle\left\{\delta \hat{f}_{m}(t), \delta \hat{f}_{n}(t)\right\}\right\rangle$ as the first moments are always null (for clarity, we have indicated time dependences explicitly). The equation of motion for the covariance matrix $\sigma$ takes the form of a deterministic diffusion equation

$$
\dot{\sigma}(t)=A \sigma(t)+\sigma(t) A^{T}+D,
$$

where $D=2 k_{a}\left(2 M_{a}+1\right) \mathbb{I}_{a} \oplus 2 k_{b}\left(2 M_{b}+1\right) \mathbb{I}_{b}$ is the diffusion matrix with $\mathbb{I}_{j}$ the $2 \times 2$ identity matrix, which is determined by assuming the two-time correlation functions for the noise operators stated above. The stability of the solution is guaranteed by the Routh-Hurwitz test, ensuring that a unique nonequilibrium steady state described by the stationary covariance matrix $\sigma_{s} \equiv \sigma(\infty)$ satisfying the Lyapunov 
equation $A \sigma_{s}+\sigma_{s} A^{T}=-D$ is eventually achieved [43]. If the whole system is noninteracting, each ensemble will equilibrate with its own thermal bath, thus leading to a final thermal state.

It is important to remark that we consider the initial covariance matrix of the composite thermometer in the so-called canonical form [44]

$$
\sigma(0)=\left(\begin{array}{ll}
\boldsymbol{\alpha} & \boldsymbol{\gamma} \\
\boldsymbol{\gamma} & \boldsymbol{\beta}
\end{array}\right)
$$

with $\boldsymbol{\alpha}=\alpha \mathbb{1}, \boldsymbol{\beta}=\beta \mathbb{1}(\alpha, \beta \geqslant 1)$, and $\boldsymbol{\gamma}=\operatorname{diag}\left[\gamma_{+}, \gamma_{-}\right]$. The entries $\alpha, \beta$, and $\gamma_{ \pm}$are bound to satisfy the conditions set for a physically legitimate covariance matrix [36], which are briefly discussed in Appendix C. Equation (5) encompasses several examples of experimentally relevant Gaussian states, such as thermal states and squeezed thermal states. Remarkably, the dynamics we consider leaves $\sigma(t)$ in canonical form throughout the evolution. In what follows, we will exploit this model to explore the readiness of the composite Gaussian probe by introducing a geometric figure of merit able to define its dynamical speed. Moreover, we seek to investigate the metrological precision of such a nonequilibrium thermometer.

\section{GEOMETRIC CONSIDERATIONS}

Here we highlight the geometric features of the dynamics at hand making use of Riemannian tools. Specifically, we refer to the set of density matrices of a given quantum system, which form a Riemannian manifold $\mathcal{S}$ over the Hilbert space $\mathcal{H}$ of the system: The one-to-one correspondence between the state of the system and its placement onto the manifold is such that, while evolving, the state will draw a curve $\ell_{\gamma}$ on $\mathcal{S}$, depending on $t$. For each $q \in \ell_{\gamma}$, an inner product on the tangent space $\mathbf{T}_{q} \mathcal{S}$ of the manifold $\mathcal{S}$ can be defined permitting the definition of an infinitesimal length $d s^{2}=Z_{\hat{\rho}}(d \hat{\rho}, d \hat{\rho})$ $[33,45,46]$. The reconstruction of an analytic form for the Riemannian metric $Z_{\hat{\rho}}$ in our problem is made difficult by the lack of a version of the Morozova-Čencov-Petz (MCP) theorem for infinite-dimensional systems [47-50]. The MCP theorem states that each Riemannian metric for discrete-variable systems is characterized by a correspondence with a set of functions that, by satisfying very restrictive conditions, can lead unambiguously to the quantum Fisher information metric $[51,52]$. The restriction to Gaussian states, however, greatly simplifies the problem as the first and second moments of the system are the only elements to be considered for its complete description $[53,54]$.

Here we focus on the Bures metric $d s_{B}^{2}=2[1-\mathcal{F}(\hat{\rho}, \hat{\rho}+$ $d \hat{\rho})$ ], which can be computed through the Uhlmann fidelity $\mathcal{F}$ between two infinitesimally close Gaussian states $\hat{\rho}$ and $\hat{\rho}+d \hat{\rho}[50,55,56]$. By calling $r$ and $\sigma$ the first and second moments of the state $\hat{\rho}$, the Bures metric can be expressed as $d s^{2}=\left(d r^{T} \sigma^{-1} d r\right) / 2+\delta / 8$, where $\delta=$ $\operatorname{Tr}\left[\mathrm{d} \sigma\left(\mathcal{L}_{\sigma}+\mathcal{L}_{\Omega}\right)^{-1} \mathrm{~d} \sigma\right]$, with $\Omega$ the $d$-mode symplectic matrix $\Omega=\oplus_{j=1}^{d} i \sigma_{y, j}$ (here $d=2$ as we consider two-mode systems and $\sigma_{y, j}$ is the $y$ Pauli matrix of the subsystem $j$ ) and $\mathcal{L}_{Y} X:=Y X Y$ for any pair of operators $X$ and $Y$. The inverse operation on the superoperator refers to the Moore-Penrose pseudoinverse.
The canonical form of the covariance matrices in our study, such as the one in Eq. (5), allows us to write the Bures metric as

$$
d s_{B}^{2}=\frac{1}{4} \sum_{j= \pm} \frac{\left[v_{j}(t+d t)-v_{j}(t)\right]^{2}}{v_{j}(t)^{2}-1},
$$

where $v_{ \pm}$represent the symplectic eigenvalues of the Gaussian state. Equation (6) provides a simple and general way to manipulate the geometrical features of the Gaussian dynamics considered [56]. As a direct consequence of Williamson's theorem, the covariance matrix of the subsystem $\sigma_{a, b}$ can be reduced in its standard form such that det $\sigma_{a, b}=v_{a, b}^{2}$, with $v_{a, b}$ the symplectic eigenvalue of the subsystem. In this way, the local form of the infinitesimal length $d s_{B, j}^{2}$ for the subsystem $j=a, b$ can be obtained as $d s_{B, j}^{2}=\frac{1}{4} \frac{\left[v_{j}(t+d t)-v_{j}(t)\right]^{2}}{v_{j}(t)^{2}-1}$, proving that $d s_{B}^{2} \neq d s_{B, a}^{2}+d s_{B, b}^{2}$.

The above constructions lead to the instantaneous speed of quantum evolution on the Riemannian manifold

$$
v_{B}^{2}(t)=\frac{1}{4} \sum_{j= \pm} \frac{\left[\partial_{t} v_{j}(t)\right]^{2}}{v_{j}(t)^{2}-1},
$$

depending on the time derivatives of the symplectic eigenvalues $v_{ \pm}$of the Gaussian state. The form of the Riemannian speed in Eq. (7) holds when setting to zero the initial value of the first moments of the quadratures of the system. It is worth stressing that, under this condition, Eq. (7) does not depend on the specific dynamics at hand and it is hence valid for closed and open system dynamics. Further considerations on the Bures metric and Riemannian speed are given in Appendix B.

In what follows, we shed light on the consequences of nonclassical correlations on the instantaneous Riemannian speed of the system. Since the latter is a purely dynamical figure of merit, it is not explicitly connected to the precision of the thermometer at hand. On one hand, such an inspection can be useful in unearthing dynamical aspects of quantum correlations, i.e., entanglement, while on the other it can lead to a more profound understanding of the dynamical readiness in the context of quantum thermometry. This exploration can be opportunely managed numerically, as correlation quantifiers are nonlinear functions of the state. In this regard, we proceed by randomly generating a large number of Gaussian states to explore the role of nonclassical features in the initial Riemannian speed of the system.

The results of our numerical analysis are shown in Fig. 2, where we consider the symmetric case of $M_{a, b}=M$. There we report the Riemannian speed of Gaussian states undergoing the dynamics shown in Eq. (4) for an arbitrarily small time $\epsilon$ versus the smallest symplectic eigenvalue $\tilde{v}_{-}$of the covariance matrix associated with the partially transposed state of the system. According to the continuous-variable (CV) counterpart of the Peres-Horodecki criterion, entangled (separable) states have $\tilde{v}_{-}<1\left(\tilde{v}_{-} \geqslant 1\right)$ states [57]. In contrast to what emerges from qubit-based thermometry [33], the thermometric phenomenology of $\mathrm{CV}$ entangled states is different from that resulting from the use of separable states. In particular, the trends displayed by Fig. 2 suggest that larger degrees of entanglement, i.e., smaller values of $\tilde{v}_{-}$, correspond to growing 

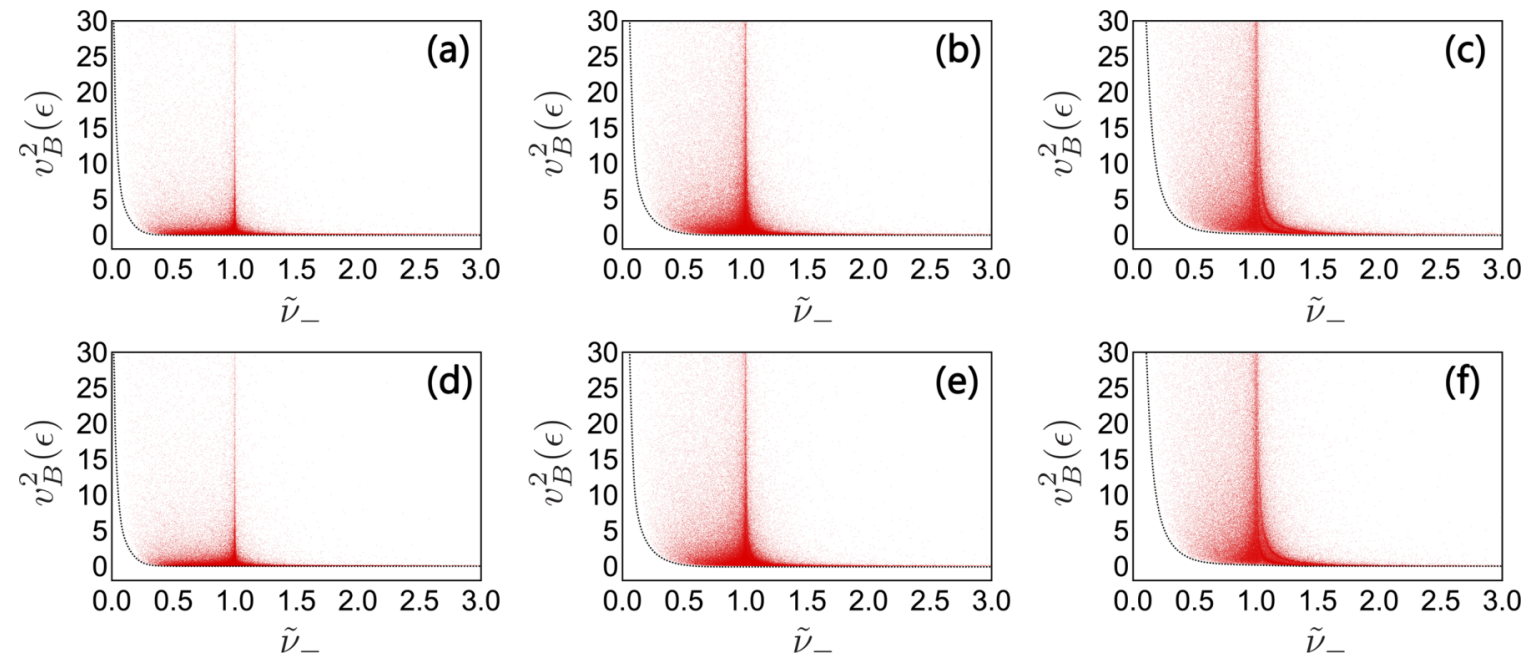

FIG. 2. Behavior of the Riemannian speed $v_{B}^{2}(\epsilon)$ vs the symplectic eigenvalue $\tilde{v}_{-}$. The panels refer to different configurations of the system, in particular, (a)-(c) $n_{G}=0$, (d)-(f) $n_{G}=0.35 \omega_{b}$, (a) and (d) $M_{a}=M_{b}=0.1$, (b) and (e) $M_{a}=M_{b}=0.5$, and (c) and (f) $M_{a}=M_{b}=1$, where $\omega_{a}=\omega_{b}=2, k_{a}=0.2 \omega_{a}$, and $k_{b}=0.1 \omega_{b}$. In the $\tilde{v}_{-} \leqslant 1$ entangled region, the lower bound on the Riemannian speed increases more and more evidently when the temperature of the baths increases.

values of the minimum initial Riemannian speed. Statistically, entangled states appear to increase the initial readiness of the thermometer. Differently from qubit-based thermometry, the physical origin of this feature seems to be intrinsically related to nonclassical effects. The numerical analysis points out the dependence of the lower bounds to the temperature of the external environment: In the entangled region, the larger the temperature of the baths, the larger the minimal initial Riemannian speed of the thermometer. Moreover, it is remarkable to highlight that maximally entangled Gaussian states at fixed global and local purities approach the lower bound to the Riemannian speed at a given degree of entanglement, thus having the slowest response to the dynamics induced by the baths. Such states can be expressed as entangled two-mode squeezed thermal states, whose free parameters can be suitably minimized to numerically build the lower bound shown in Fig. 2. The presence of the interaction $(G \neq 0)$ between the two subsystems does not affect the dynamical behavior of the thermometer in Fig. 2 as it is expected to emerge at longer times.

\section{METROLOGICAL CONSIDERATIONS}

As a second point of our investigation, we aim at exploiting the designed setup to build indirect measurement schemes able to infer temperature-related parameters associated with the environment. As we are probing a property of the bath, which is maintained in its initial thermal state, the temperature here is a well-defined parameter throughout the evolution. In other words, we are not concerned with an effective temperature of the probe, nor do we need to be. In particular, the case at hand is ideal for this purpose as the adopted system can be initialized with a high degree of control and then effectively measured after its interaction with the sample to be probed. In the adopted scheme, we consider $\mathcal{N}$ independent copies of the system at hand, allowing for the same number of independent measurements which can be implemented to obtain an unbiased estimator $\tilde{M}$ for the average number of excitations in the baths $M$. In this case, the variance $\Delta^{2} M=\left\langle(\tilde{M}-M)^{2}\right\rangle$ satis- fies the quantum Cramér-Rao bound $\Delta^{2} M \geqslant 1 / \mathcal{N} Q_{M}$, where $Q_{M}$ is the quantum Fisher information. Plainly, the precision of the measurement can be enhanced by increasing the QFI [58-60]. The latter can be interpreted as the distance between Gaussian states interacting with thermal baths whose average numbers of excitations differ by an infinitesimal variation, so $Q_{M}(t)=8\left\{1-\mathcal{F}\left[\hat{\rho}_{M}(t), \hat{\rho}_{M+d M}(t)\right]\right\} / d M^{2}$, where the functional $\mathcal{F}$ is the Uhlmann fidelity between the Gaussian states of the thermometer $\hat{\rho}_{M}$ and $\hat{\rho}_{M+d M}$ which are interacting with environments at temperatures $M$ and $M+d M$, respectively. Remarkably for Gaussian states, the evaluation of the QFI can be traced back to the Wigner formalism since the first and second moments are sufficient for the complete description of the state [56]. In the specific case at hand, where the initial first moments have been set to zero, the fidelity can be defined as

$$
\mathcal{F}^{4}\left[\hat{\rho}_{M}(t), \hat{\rho}_{M+d M}(t)\right]=\frac{\operatorname{det}\left[2\left(\sqrt{\mathbb{I}+\frac{\left[\Xi ( t ) \Omega \left[^{-2}\right.\right.}{4}}+\mathbb{I}\right) \Xi(t)\right]}{\operatorname{det}\left[\frac{\sigma_{M}(t)+\sigma_{M+d M}(t)}{2}\right]},
$$

where $2 \Omega \Xi(t)=\left[\sigma_{M}(t)+\sigma_{M+d M}(t)\right]^{-1}\left[\Omega+\sigma_{M+d M}(t) \Omega \sigma_{M}\right.$ $(t)$.

A reparametrization of the QFI is made possible by the fact that $M$ is a continuously differentiable function of $T$. In this spirit, the QFI on the temperature can be expressed as

$$
Q_{T}(t)=Q_{M}(t) \frac{\omega^{2} \operatorname{csch}^{4}\left(\frac{\omega}{2 T}\right)}{16 T^{4}} .
$$

The plots in Fig. 3 show the dispersion of the nonequilibrium QFI $Q_{M}(t)$ as a function of the interaction time $t$. At variance with the findings in Refs. [9,14,18] where qubitbased thermometers have been considered, we notice how nonequilibrium conditions do not enhance the precision of temperature estimation in the Gaussian regime. In particular, $Q_{M}(t)$ reaches its maximum value when the thermometer approaches its steady state. Figure 3 shows that thermometers 

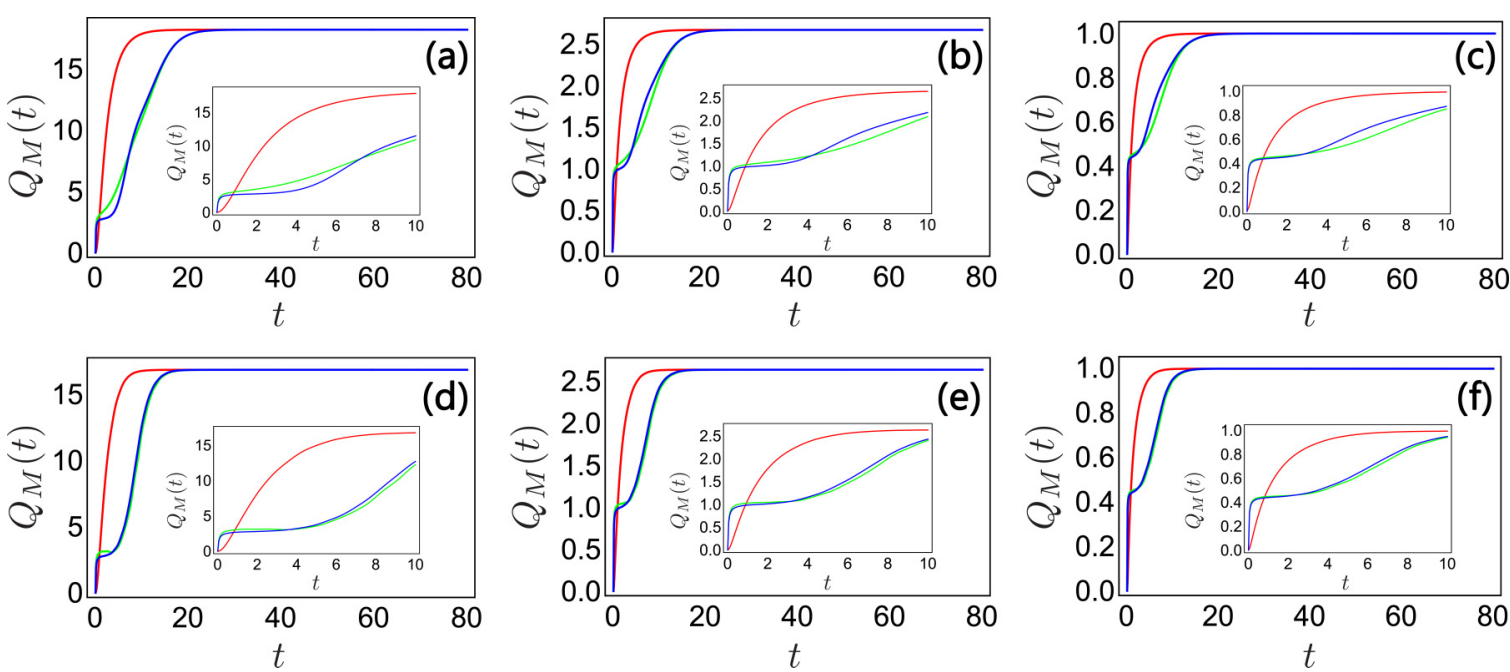

FIG. 3. Plots of the nonequilibrium QFI $Q_{M}(t)$ for different initial preparations of the system as a function of the interaction time $t$. In all plots, red curves describe the behavior of $Q_{M}(t)$ for initial thermal states $\sigma_{0}=\left(1+2 \bar{M}_{a}\right) \mathbb{I}_{2} \oplus\left(1+2 \bar{M}_{b}\right) \mathbb{I}_{2}$ with $\bar{M}_{a}=\bar{M}_{b}=0.1$, blue curves show the same dispersion for locally squeezed vacuum states $\sigma_{0}=\left(S_{a} \sigma_{0, a} S_{a}^{T}\right) \oplus\left(S_{b} \sigma_{0, b} S_{b}^{T}\right)$ with $\sigma_{0, a}=\sigma_{0, b}=\mathbb{I}_{2}$ and $S_{a}\left(S_{b}\right)$ a squeezing transformation with the parameter $r_{a}=2\left(r_{b}=-2\right)$, and green curves are associated with the initial two-mode squeezed states (twin-beam states) with the parameter $r=2$. The maximum of the QFI is reached when the system approaches its steady state, so any absolute metrological advantage in the nonequilibrium regime is not observed. At the very beginning of the dynamics, the squeezed states considered show an advantage over their thermal counterpart: The rung characterizing such an advantage is more pronounced when the temperature of the bath is higher [(a) and (d) $M=0.1$, (b) and (e) $M=0.5$, and (c) and (f) $M=1$ ]. The presence of the interaction between the two ensembles does not modify the general trend of the QFI $\left[(\mathrm{a})-(\mathrm{c}) n_{G}=0\right.$ and (d)-(f) $\left.n_{G}=0.35 \omega_{b}\right]$. The parameters of the dynamics are $\omega_{a}=\omega_{b}=2, k_{a}=0.2 \omega_{a}$, and $k_{b}=0.1 \omega_{b}$.

prepared in a thermal state allow one to get the maximum of the QFI in less time if compared to initial locally squeezed or two-mode squeezed states. The presence of nonclassical resources in the initial state of the thermometer brakes the achievement of the maximum in the QFI, but powers up its precision for very short times. Entanglement does not appear to have a clear role in enhancing the performance of the probe: Indeed, thermometers prepared in two-mode squeezed states can lead to both increased and decreased metrological performances if compared to those prepared in locally squeezed vacuum states. In particular, the quantum enhancement in temperature estimation due to the presence of the nonclassical correlation is well displayed at short times, where entanglement leads up the QFI. It is remarkable to highlight that such an advantage appears more pronounced for low temperatures of the external thermal bath and deteriorates the precision of the thermometer in the remaining transient of the nonequilibrium dynamics. The presence of the interaction between the subsystems accelerates the attainment of the optimal metrological condition and reduces the gap between two-mode squeezed states and locally squeezed vacuum states.

\section{CONCLUSION}

We have shown that the link between quantum features and facets of quantum thermometry are often elusive. By addressing explicitly the CV regime, we have highlighted a profound discrepancy in the way small-scale thermometers are influenced by quantum features. In the case examined, the instantaneous response of the Gaussian thermometer to the interaction with the environment shows a dependence on nonclassical correlations among probing subsystems: The minimum initial dynamical speed of the system increases with the amount of entanglement and depends on the temperature of the bath. There are no exceptions for the performance of the thermometer, which can be powered up for very short times by using quantum resources. The latter, however, can be detrimental for the remainder transient dynamics of the thermometer, reducing the nonequilibrium QFI with respect to classical states. The picture coming out of our assessment pushes for further exploration of the role of quantum facets in quantum thermometry. The presence of higher-order nonlinearities in the interaction Hamiltonian of the subsystems ought to be taken in consideration to investigate unexplored thermometric regimes.

Note added. Recently, we became aware of a related work in preparation by I. Gianani et al. [61] discussing about the discrimination of thermal baths by single qubit probes.

\section{ACKNOWLEDGMENTS}

The authors would like to thank Antonella De Pasquale, Gabriele De Chiara, Gabriel Landi, Obinna Abah, Michele Maria Feyles, and Federico Centrone for stimulating discussions. L.M. acknowledges the hospitality of Università degli Studi Roma Tre, where part of this work was carried out. The authors acknowledge financial support from H2020-FETOPEN-2018-2020 project TEQ (Grant No. 766900), the DfE-SFI Investigator Programme (Grant No. 15/IA/2864), COST Action CA15220 and UK ESPRC (No. EP/T028106/1), the Royal Society Wolfson Research Fellowship (Grant No. RSWF\R3\183013), the Royal Society International Exchanges Programme (Grant No. IEC\R2\192220), the Leverhulme Trust Research Project Grant (Grant No. 
RGP-2018-266), the UK EPSRC (No. EP/T028106/1), the Angelo Della Riccia Foundation (Royal Decree 19.7.41. No. 979, Florence), and MIUR, Rita Levi-Montalcini Fellowship.

\section{APPENDIX A: HOLSTEIN-PRIMAKOFF TRANSFORMATION}

In this Appendix we briefly show how to manipulate the Hamiltonian of the whole system through the HolsteinPrimakoff transformation.

Assuming that $N \gg 1$, the Holstein-Primakoff transformation to boson creation and annihilation operators is defined by these relations

$$
\hat{S}_{j}^{+}=\sqrt{N} \hat{j}^{\dagger} \hat{J}, \quad \hat{S}_{j}^{-}=\sqrt{N} \hat{J} \hat{j}, \quad \hat{S}_{j}^{z}=\hat{j}^{\dagger} \hat{j}-\frac{N}{2},
$$

where $\left[\hat{j}, \hat{j}^{\dagger}\right]=1$ and $\hat{J}=\sqrt{1-\hat{j}^{\dagger} \hat{j} / N}$ ( $\hat{A}$ and $\hat{B}$ for the considered ensembles) is an operator ensuring that the operators in Eq. (A1) fulfill the SU(2) algebra. It is fair to say that the operators in Eq. (A1) can be referred to the specific ensemble of atomic spin states by substituting $\hat{a}$ and $\hat{b}$ for the generic operator $\hat{j}$. In terms of the collective bosonic Dicke operators, the Hamiltonian of the system $\hat{H}_{H P}$ results in the sum of the free Hamiltonian $\hat{H}_{H P}^{0}=\hbar \omega_{a}\left[\hat{a}^{\dagger} \hat{a}-(N-\right.$ 1)/2] $+\hbar \omega_{b}\left[\hat{b}^{\dagger} \hat{b}-(N-1) / 2\right]$ and the interaction one $\hat{H}_{H P}^{\text {int }}=$ $\hbar G / 2\left(\sqrt{N} \hat{a}^{\dagger} \hat{A}+\sqrt{N} \hat{A} \hat{a}\right)\left(\sqrt{N} \hat{b}^{\dagger} \hat{B}+\sqrt{N} \hat{B} \hat{b}\right)$. The presence or not of nonlinear effects from the $\hat{J}$-like operators results from a trade-off between $\left\langle\hat{j}^{\dagger} \hat{j}\right\rangle$ and $N$. In general, the operators in Eq. (A1) can be manipulated through the $\hat{J}=$ $\sum_{h=0}^{\infty} \frac{(2 h) !}{(1-2 h)\left(2^{h} h !\right)} \frac{\left(\hat{j}^{\dagger} \hat{j}\right)^{h}}{N^{h}}$ expansion: Here we limit the expansion to $\hat{J} \sim 1-\hat{j}^{\dagger} \hat{j} / 2 N+O\left(N^{-2}\right)$, which leads to the nonlinear interaction Hamiltonian $\hat{H}_{H P}^{\text {int }}=\hbar n_{G} / 2\left(\hat{a}^{\dagger}-\hat{a}^{\dagger} \hat{a}^{\dagger} \hat{a} / 2 N+\right.$ H.c.) $\left(\hat{b}^{\dagger}-\hat{b}^{\dagger} \hat{b}^{\dagger} \hat{b} / 2 N+\right.$ H.c. $)$, corresponding to the Hamiltonian used in the main text. It is remarkable to verify that the structure of this interaction Hamiltonian reduces to the one describing a positionlike interaction between harmonic oscillators when dropping the nonlinear contributions from the expansion.

\section{APPENDIX B: GEOMETRIC CONSIDERATIONS: BURES DISTANCE}

The Bures distance between two arbitrary Gaussian states with density matrices $\rho_{1}$ and $\rho_{2}$ is given by $D_{B}\left(\rho_{1}, \rho_{2}\right)=$ $2\left(1-\mathcal{F}\left[\rho_{1}, \rho_{2}\right]\right)$. By expanding the fidelity, we can derive the Bures metric. When dealing with two infinitesimally close Gaussian states $\rho_{1}=\rho$, with covariance matrix $\sigma$, and $\rho_{2}=$ $\rho+d \rho$, with covariance matrix $\sigma+d \sigma$, the Bures metric is given by $d s_{B}^{2}=2(1-\mathcal{F}[\hat{\rho}, \hat{\rho}+d \hat{\rho}])$. When the initial first statistical moments are null, the Bures metric reduces to $d s_{B}^{2}=\delta / 8$ [56] where, for manifolds of quantum states characterized by a covariance matrix written in canonical form [44] such as the one we are considering in this work [cf., Eq. (5)], we have

$$
\delta=\sum_{i j} \delta_{i j}^{K} \frac{d w_{i} d w_{j}}{w_{i} w_{j}-1}
$$

with $\delta_{i j}^{K}$ denoting the Kronecker delta, $w_{i}$ representing the $i$ th eigenvalues of $W=-i \sigma \Omega$, and $d w_{i}$ representing its infinitesimal change. This implies Eq. (6), with which we can achieve the form of the Riemannian speed in Eq. (7). A more compact, but equivalent, form of the Riemannian speed can be obtained by deforming the simplex, thus introducing the coordinate

$$
\Gamma_{i}=\frac{1}{4 \sqrt{2}} \ln \left(\frac{\sqrt{w_{i} w_{i}-1}+w_{i}}{\sqrt{w_{i} w_{i}-1}-w_{i}}\right)
$$

such that $d \Gamma_{i}=\left(d \Gamma_{\alpha} / d w_{\alpha}\right) d w_{i}$, with $\quad d \Gamma_{\alpha} / d w_{\alpha}=$ $\left(2 \sqrt{2} \sqrt{w_{\alpha} w_{\alpha}-1}\right)^{-1}$. Under this deformation, $d s_{B}^{2}=$ $\sum_{i} d \Gamma_{i} d \Gamma_{i}$, so $v_{B}^{2}(t)=\sum_{i} \frac{d \Gamma_{i}}{d t} \frac{d \Gamma_{i}}{d t}$.

\section{APPENDIX C: GENERATION OF RANDOM GAUSSIAN STATES}

Any physically legitimate covariance matrix $\sigma$ should satisfy the condition of positivity $(\sigma \geqslant 0)$ as well as the Robertson-Schrödinger uncertainty relation $(\sigma+i \Omega) \geqslant 0$. The entries of $\sigma$ can be cast in terms of the purity of the marginal state of each subsystem $\mu_{\zeta}=(\operatorname{det} \zeta)^{-1 / 2}=1 / \zeta \in$ $(0,1]$, the global purity $\mu=(\operatorname{det} \sigma)^{-1 / 2}=\left[\left(\alpha \beta-\gamma_{+}^{2}\right)(\alpha \beta-\right.$ $\left.\left.\gamma_{-}^{2}\right)\right]^{-1 / 2} \in(0,1]$, and a further symplectic invariant (the socalled seralian) defined as $\Delta=\alpha^{2}+\beta^{2}+2 \gamma_{+} \gamma_{-}$, which is constrained as $2 / \mu+\left(\mu_{\alpha}-\mu_{\beta}\right)^{2} / \mu_{\alpha}^{2} \mu_{\beta}^{2} \leqslant \Delta \leqslant \min \left\{\left(\mu_{\alpha}+\right.\right.$ $\left.\left.\mu_{\beta}\right)^{2} / \mu_{\alpha}^{2} \mu_{\beta}^{2}-(2 / \mu), 1+\left(1 / \mu^{2}\right)\right\}$. The global purity and the seralian are invariant under generic symplectic transformations, while the local purities are invariant only for transformations acting independently on the two modes. It is straightforward to check that in terms of such quantities we have $\gamma_{ \pm}=\sqrt{\mu_{\alpha} \mu_{\beta}}\left(\eta_{-} \mp \eta_{+}\right) / 4$, with $\eta_{\mp}=$ $\sqrt{\left[\Delta-\left(\mu_{\alpha} \mp \mu_{\beta}\right)^{2} / \mu_{\alpha}^{2} \mu_{\beta}^{2}\right]^{2}-4 / \mu^{2}}$.

According to the $\mathrm{CV}$ version of the Peres-Horodecki criterion for entanglement [57], the bipartite Gaussian state corresponding to $\sigma$ is entangled if and only if $\tilde{v}_{-}<1$, where $\tilde{v}_{-}$is the smallest symplectic eigenvalue of the covariance matrix associated with the partially transposed state of the system, reading $\nu_{-}=\left(\tilde{\Delta}-\sqrt{\tilde{\Delta}^{2}-4 / \mu^{2}}\right)^{1 / 2} / \sqrt{2}$, with $\tilde{\Delta}=\Delta-4 \operatorname{det} \gamma$. This happens for $\tilde{\Delta} \geqslant 1+1 / \mu^{2}$.

By sampling the values of $\mu_{\alpha, \beta}$ and $\mu$ within their respective ranges, one can generate physical (separable or entangled) Gaussian states. States such that $\mu_{\alpha} \mu_{\beta} \leqslant \mu \leqslant$ $\mu_{\alpha} \mu_{\beta} /\left(\mu_{\alpha}+\mu_{\beta}-\mu_{\alpha} \mu_{\beta}\right)$ belong to the separability region. Separable and entangled states coexist for choices of local and global purities such that $\mu_{\alpha} \mu_{\beta} /\left(\mu_{\alpha}+\mu_{\beta}-\mu_{\alpha} \mu_{\beta}\right)<\mu \leqslant$ $\mu_{\alpha} \mu_{\beta} / \sqrt{\mu_{\alpha}^{2}+\mu_{\beta}^{2}-\mu_{\alpha}^{2} \mu_{\beta}^{2}}$, while inseparability is guaranteed for the remaining (physically allowed) values of the global purity.

When fixing both global and local purities, Gaussian maximally entangled states can be obtained by choosing $\Delta=2 / \mu+\left(\mu_{\alpha}-\mu_{\beta}\right)^{2} / \mu_{\alpha}^{2} \mu_{\beta}^{2}$. Gaussian maximally entangled states at fixed global and local purities (GMEMS) have $\gamma_{ \pm}= \pm\left(1 / \mu_{\alpha} \mu_{\beta}-1 / \mu\right)^{1 / 2}$. In the space of entangled states, GMEMS have been shown to be the slowest ones, as they achieve the lower bound in the Riemannian speed plot in Fig. 2. 
[1] Lord Kelvin famously stated that "when you can measure what you are speaking about, and express it in numbers, you know something about it; but when you cannot measure it, when you cannot express it in numbers, your knowledge is of a meagre and unsatisfactory kind: it may be the beginning of knowledge, but you have scarcely, in your thoughts, advanced to the stage of science." W. Thomson, Popular Lectures and Addresses (Macmillan, London, 1889), Vol. 1, p. 73.

[2] K. Jacobs, Quantum Measurement Theory and its Applications (Cambridge University Press, Cambridge, 2014).

[3] L. Michalski, K. Eckersdorf, J. Kucharski, and J. McGhee, Temperature measurement, Meas. Sci. Technol. 13, 1651 (2002).

[4] F. Giazotto, T. T. Heikklä, A. Luukanen, A. M. Savin, and J. Pekola, Opportunities for mesoscopics in thermometry and refrigeration: Physics and applications, Rev. Mod. Phys. 78, 217 (2006).

[5] C. D. S. Brites, P. P. Lima, N. J. O. Silva, A. Millán, V. S. Amaral, F. Palacio, and L. D. Carlos, Thermometry at the nanoscale, Nanoscale 4, 4799 (2012).

[6] S. Razavian, C. Benedetti, M. Bina, Y. Akbari-Kourbolagh, and M. G. A. Paris, Quantum thermometry by single-qubit dephasing, Eur. Phys. J. Plus 134, 284 (2019).

[7] F. Gebbia, C. Benedetti, F. Benatti, R. Floreanini, M. Bina, and M. G. A. Paris, Two-qubit quantum probes for the temperature of an Ohmic environment, Phys. Rev. A 101, 032112 (2020).

[8] M. Brunelli, S. Olivares, and M. G. A. Paris, Qubit thermometry for micromechanical resonators, Phys. Rev. A 84, 032105 (2011).

[9] S. Jevtic, D. Newman, T. Rudolph, and T. M. Stace, Singlequbit thermometry, Phys. Rev. A 91, 012331 (2015).

[10] M. G. A. Paris, Achieving the Landau bound to precision of quantum thermometry in systems with vanishing gap, J. Phys. A: Math. Theor. 49, 03LT02 (2015).

[11] L. Seveso and M. G. A. Paris, Trade-off between information and disturbance in qubit thermometry, Phys. Rev. A 97, 032129 (2018).

[12] T. L. Hill, A different approach to nanothermodynamics, Nano Lett. 1, 273 (2001).

[13] A. De Pasquale, D. Rossini, R. Fazio, and V. Giovannetti, Local quantum thermal susceptibility, Nat. Commun. 7, 12782 (2016).

[14] V. Cavina, L. Mancino, A. De Pasquale, I. Gianani, M. Sbroscia, R. I. Booth, E. Roccia, R. Raimondi, V. Giovannetti, and M. Barbieri, Bridging thermodynamics and metrology in nonequilibrium quantum thermometry, Phys. Rev. A 98, 050101(R) (2018).

[15] S. Deffner and E. Lutz, Nonequilibrium Entropy Production for Open Quantum Systems, Phys. Rev. Lett. 107, 140404 (2011).

[16] L. Mancino, V. Cavina, A. De Pasquale, M. Sbroscia, R. I. Booth, E. Roccia, I. Gianani, V. Giovannetti, and M. Barbieri, Geometrical Bounds on Irreversibility in Open Quantum Systems, Phys. Rev. Lett. 121, 160602 (2018).

[17] L. Mancino, M. Sbroscia, E. Roccia, I. Gianani, F. Somma, P. Mataloni, M. Paternostro, and M. Barbieri, The entropic cost of quantum generalised measurements, npj Quantum Inf. 4, 20 (2018).

[18] L. Mancino, M. Sbroscia, I. Gianani, E. Roccia, and M. Barbieri, Quantum Simulation of Single-Qubit Thermometry Using Linear Optics, Phys. Rev. Lett. 118, 130502 (2017).

[19] T. M. Stace, Quantum limits of thermometry, Phys. Rev. A 82, 011611(R) (2010).
[20] P. Neumann, I. Jakobi, F. Dolde, C. Burk, R. Reuter, G. Waldherr, J. Honert, T. Wolf, A. Brunner, J. H. Shim, D. Suter, H. Sumiya, J. Isoya, and J. Wrachtrup, High-precision nanoscale temperature sensing using single defects in diamond, Nano Lett. 13, 2738 (2013).

[21] R. Kosloff, Quantum thermodynamics: A dynamical viewpoint, Entropy 15, 2100 (2013).

[22] G. Kucsko, P. C. Maurer, N. Y. Yao, M. Kubo, H. J. Noh, P. K. Lo, H. Park, and M. D. Lukin, Nanometre-scale thermometry in a living cell, Nature (London) 500, 54 (2013).

[23] L. A. Correa, M. Mehboudi, G. Adesso, and A. Sanpera, Individual Quantum Probes for Optimal Thermometry, Phys. Rev. Lett. 114, 220405 (2015).

[24] S. Campbell, M. Mehboudi, G. De Chiara, and M. Paternostro, Global and local thermometry schemes in coupled quantum systems, New J. Phys. 19, 103003 (2017).

[25] S. Campbell, M. G. Genoni, and S. Deffner, Precision thermometry and the quantum speed limit, Quantum Sci. Technol. 3, 025002 (2018).

[26] M. Sbroscia, I. Gianani, L. Mancino, E. Roccia, Z. Huang, L. Maccone, C. Macchiavello, and M. Barbieri, Experimental ancilla-assisted phase estimation in a noisy channel, Phys. Rev. A 97, 032305 (2018).

[27] S. Seah, S. Nimmrichter, D. Grimmer, J. P. Santos, V. Scarani, and G. T. Landi, Collisional Quantum Thermometry, Phys. Rev. Lett. 123, 180602 (2019).

[28] D. Farina, V. Cavina, and V. Giovannetti, Quantum bath statistical tagging, Phys. Rev. A 100, 042327 (2019).

[29] M. R. Jørgensen, P. P. Potts, M. G. A. Paris, and J. B. Brask, Tight bound on finite-resolution quantum thermometry at low temperatures, Phys. Rev. Res. 2, 033394 (2020).

[30] A. Monras and F. Illuminati, Measurement of damping and temperature: Precision bounds in Gaussian dissipative channels, Phys. Rev. A 83, 012315 (2011).

[31] O. Pinel, P. Jian, N. Treps, C. Fabre, and D. Braun, Quantum parameter estimation using general single-mode Gaussian states, Phys. Rev. A 88, 040102 (2013).

[32] Y. Gao and H. Lee, Bounds on quantum multiple-parameter estimation with Gaussian state, Eur. Phys. J. D 68, 347 (2014).

[33] M. M. Feyles, L. Mancino, M. Sbroscia, I. Gianani, and M. Barbieri, Dynamical role of quantum signatures in quantum thermometry, Phys. Rev. A 99, 062114 (2019).

[34] C. Weedbrook, S. Pirandola, R. García-Patrón, N. J. Cerf, T. C. Ralph, J. H. Shapiro, and S. Lloyd, Gaussian quantum information, Rev. Mod. Phys. 84, 621 (2012).

[35] A. Ferraro, S. Olivares, and M. G. A. Paris, Gaussian States in Continuous Variable Quantum Information (Bibliopolis, Naples, 2005).

[36] A. Serafini, Quantum Continuous Variables: A Primer of Theoretical Methods (Taylor \& Francis, Oxford, 2017).

[37] R. Nichols, P. Liuzzo-Scorpo, P. A. Knott, and G. Adesso, Multiparameter Gaussian quantum metrology, Phys. Rev. A 98, 012114 (2018).

[38] K. Hammerer, A. S. Sørensen, and E. Polzik, Quantum interface between light and atomic ensembles, Rev. Mod. Phys. 82, 1041 (2010).

[39] H.-P. Breuer and F. Petruccione, The Theory of Open Quantum Systems (Oxford University Press, New York, 2002). 
[40] T. Holstein and H. Primakoff, Field dependence of the intrinsic domain magnetization of a ferromagnet, Phys. Rev. 58, 1098 (1940).

[41] M. Marcuzzi, J. Marino, A. Gambassi, and A. Silva, Prethermalization from a low-density Holstein-Primakoff expansion, Phys. Rev. B 94, 214304 (2016).

[42] M. Paternostro, S. Gigan, M. S. Kim, F. Blaser, H. R. Böhm, and M. Aspelmeyer, Reconstructing the dynamics of a movable mirror in a detuned optical cavity, New J. Phys. 8, 107 (2006).

[43] A. Hurwitz, in Selected Papers on Mathematical Trends in Control Theory, edited by R. Bellman and R. Kalaba (Dover, New York, 1964).

[44] R. Simon, Peres-Horodecki Separability Criterion for Continuous Variable Systems, Phys. Rev. Lett. 84, 2726 (2000).

[45] I. Bengtsson and K. Zyczkowski, Geometry of Quantum States: An Introduction to Quantum Entanglement (Cambridge University Press, Cambridge, 2006).

[46] D. P. Pires, M. Cianciaruso, L. C. Céleri, G. Adesso, and D. O. Soares-Pinto, Generalized Geometric Quantum Speed Limits, Phys. Rev. X 6, 021031 (2016).

[47] E. A. Morozova and N. N. Cëncov, Markov invariant geometry on manifolds of states, J. Sov. Math. 56, 2648 (1991).

[48] D. Petz and H. Hasegawa, On the Riemannian metric of $\alpha$-entropies of density matrices, Lett. Math. Phys. 38, 221 (1996).

[49] D. Petz, Monotone metrics on matrix spaces, Linear Algebra Appl. 244, 81 (1996).
[50] D. Petz, Covariance and Fisher information in quantum mechanics, J. Phys. A: Math. Gen. 35, 929 (2002).

[51] A. Uhlmann, Density operators as an arena for differential geometry, Rep. Math. Phys. 33, 253 (1993).

[52] A. Uhlmann, Geometric phases and related structures, Rep. Math. Phys. 36, 461 (1995).

[53] A. Monras and F. Illuminati, Information geometry of Gaussian channels, Phys. Rev. A 81, 062326 (2010).

[54] K. Siudzińska, K. Luoma, and W. T. Strunz, Geometry on the manifold of Gaussian quantum channels, Phys. Rev. A 100, 062308 (2019).

[55] D. Petz and C. Sudár, Geometries of quantum states, J. Math. Phys. 37, 2662 (1996).

[56] L. Banchi, S. L. Braunstein, and S. Pirandola, Quantum Fidelity for Arbitrary Gaussian States, Phys. Rev. Lett. 115, 260501 (2015).

[57] G. Giedke, B. Kraus, M. Lewenstein, and J. I. Cirac, Entanglement Criteria for All Bipartite Gaussian States, Phys. Rev. Lett. 87, 167904 (2001).

[58] V. Giovannetti, S. Lloyd, and L. Maccone, Quantum-enhanced measurements: Beating the standard quantum limit, Science 306, 1330 (2004).

[59] V. Giovannetti, S. Lloyd, and L. Maccone, Quantum Metrology, Phys. Rev. Lett. 96, 010401 (2006).

[60] S. Pirandola, B. R. Bardhan, T. Gehring, C. Weedbrook, and S. Lloyd, Advances in photonic quantum sensing, Nat. Photon. 12, 724 (2018).

[61] I. Gianani et al., Discrimination of thermal baths by single-qubit probes, Phys. Rev. Res. 2, 033497 (2020). 